\title{
How Does User Experience Affect Network Effects of Social Media Platforms?
}

\author{
Guanbiao Li \\ University of California, Davis, California, Davis, 95616, United States of America \\ Email:1010956541@qq.com
}

\begin{abstract}
The success of social media platforms depends significantly on whether it is able to create large network effects such that advertisers and businesses are attracted to their platforms. The sole purpose of social media platforms is to get people to interact through different types of communication, including text chat, video chat, image, post, etc. How comfortable users are when they use a social media platform decides whether they will stay on that platform and participate. Therefore, user decision and participation in social media platforms play an important role in determining the scale of network effects. This paper mainly focuses on how user experience influences network effects of social media platforms. User experience can be affected in many aspects, and this paper discusses aspects of demographic effects, platform functionality, user privacy, distrust and misinformation, social anxiety, and platform-based advertisements and businesses. To assess how those factors affect user experience, several surveys and data are collected from the Internet.
\end{abstract}

Keywords: Network Effects, User Experience, Demographic Effects, Platform Functionality, User Privacy, Platform Distrust and Misinformation, Social Anxiety, Platform-based Advertisements and Businesses

\section{INTRODUCTION}

This paper discusses the influence of user experience on social media platforms from six aspects, which are in order platform usage by demographic effects, platform functionality, user privacy, distrust and misinformation, social anxiety suffered by platform users, and platformbased advertisements and businesses. I collected many surveys from the Internet, mainly from a renowned research website called Pew Research Center, to analyze how each aspect affects the network effects of social media platforms. Those surveys were mainly conducted in the U.S. and might be less useful in other countries and regions.

\section{DEMOGRAPHIC EFFECTS}

Users tend to use social media platforms that share one or many similar characteristics with them, therefore users can easily find their friends or other users with similar interests on platforms and obtain a sense of belonging. Demographic difference in the usage of social media platforms can help social media platform companies identify how user similarity and difference behaves. Demographic differences can have either major and minor effects on user experience, depending on the nature of social media platforms. By knowing this difference, social media platforms should know what characteristics they share with users and target this particular group of users with the majority of platform cost.

According to a Pew Research Center survey conducted from Jan. 8 to Feb, 7, 2019, the usage of social media platforms differs by age, income level, education level and many more [6]. But demographic differences vary on every social media platform. An age-related difference stood out in social media platforms such as Instagram and Snapchat, which are used substantially by the young-age people around 18- to 29-year-olds. Facebook shows less age differences in media use than Instagram and Snapchat, but a huge difference in income level. Lower-income teens are more likely than higherincome teens to use Facebook. This survey does not include juveniles, those whose age is under 18 years old [2].

People whose income is more than $\$ 75000$ used Twitter, LinkedIn and Pinterest more often than those whose income is below $\$ 75000$. Also, people who are more educated, tend to use Twitter, LinkedIn and Pinterest more often than those who are less educated. It 
is clear to see a similarity between income level and education level [6].

Income levels and education levels tell the same thing because they both represent users' lifestyle. People who are more educated tend to have more income than people who are less educated and probably spend more time on formal and commercial matters. LinkedIn, Twitter and Pinterest are more like social media platforms for business use not for people who often use social media platforms to contact with friends. Secondly, age differences show a distinct liking between different age groups of people. Majority of young people use social media platforms for fun, such as watching video, texting with friends, or playing platform-based video games. They tend to like using the social media platform like Instagram that is beautifully designed and is able to post images and catch fashion trends. The old generation generally likes to use social media platforms that are more functional. Therefore, platforms need to clearly understand the connection among age, income level and education level and also need to know whether the majority of users use the platform for fun or for business. Social media platform companies should know which demographic group they should target and build the platform around this group of users.

Same groups of users staying on the same social media platform will generate positive within-group external effects by attracting more groups of users to the platform. In this case, the large amount of newcomers will also attract advertisers and businesses because they can easily target those users by just focusing only one platform. Thus, large network effects are created and platforms make revenue from advertisers and businesses.

\section{PLATFORM FUNCTIONALITY}

People use social media platforms for different reasons. Some may just use it to contact friends, others may have more important tasks, such as helping raise an awareness for a cause or a problem in the society. Different reasons for using platforms mean that platforms need to have the functions that enable users to do all of their matters. But we need to know which function is used more frequently than others and how users are using this function.

A survey shows that $63 \%$ of users use social media platforms to keep in touch with friends, and $45 \%$ of users use it to make connections with new people [10]. Another survey shows that $87.40 \%$ of users prefer the content of personal photos and $69.83 \%$ of users prefer text messages [9]. Personal photos and text messages are both related to communication with others, which tells that social media platforms have the function that enables users to contact their friends.

A survey conducted by Pew Research Center shows that two-thirds of users get news from social media platforms. $66 \%$ of U.S. adults use Facebook, and $45 \%$ of them get news on Facebook. 15\% of U.S. adults use Twitter and $11 \%$ of them get news on Twitter [7]. This data suggests that getting news from social media platforms might not be users' primary reason for using social media platforms, but the majority of users do so.

Communicating with friends and getting news on the social media platform both suggest that social media platforms have changed most people's lifestyles and receive large positive network effects. All social media platforms have different primary functions and build minor and supplementary functions to enhance the usage of their primary one. In order to improve the service of communication, platforms should improve primary functions, such as images and text messages.

The news on social media platforms should be accurate and authentic and avoid causing polarization, which is a common phenomenon on many social media platforms. Political campaigns are often involved around the news on platforms. Platforms need to make sure they provide those news in a neutral political stand point and do not create intentional bias among users. Moreover, in order for users to talk about their thoughts after reading news, platforms should create a small platform for news sharing. It can be used to have communication about political and society improvement, which, overall, has positive effects on society development.

Platform functionality can generate either positive and negative within-groups external effects on users, depending on whether users are able to do their matters with the platform communication function. Therefore, social media platforms should constantly keep updating their primary and minor functions to let users use their service more easily and conveniently.

\section{USER PRIVACY}

User privacy greatly affects user experience because it safeguards the communication that takes place between users on the platform and also provides security on transactions that are made on social media platforms such as a transaction between a user and a platform-based business. People value user privacy differently, but it can be said that the better social media platforms regulate user privacy, the more users will come to their platforms.

A survey of user privacy on social media platform done by Edith Cowan University suggests that though social media platforms are setting tighter privacy policies and users are aware of the risk of over-disclosing private information on the platform, they are still willing to post much of their private information because they want to get a sense of belonging from their community or their friend zone. Fake profiles, identities and information do not create much caution on users [1].

User privacy only has a modest effect on network effects but still remains a huge problem for the future. 
Despite the fact that many platform users are concerned about their personal privacy, they still disclose quite a lot of information on social media platforms. But that does not mean user privacy is not a problem at all. Nowadays, most social media platforms have constantly improved their security service and have showed many changes on protecting user privacy, but the dangers such as cyber security, can easily damage a society. Therefore, social media platform companies should pay more attention to the protection of user information and avoiding information leakage.

\section{PLATFORM DISTRUST AND MISINFORMATION}

If people do not trust using something, it is more likely they will not use it for long. A survey conducted by Pew Research Center shows that $51 \%$ of U.S. users are not too confident about social media sites they use [8]. Also, another problem related to distrust is misinformation. If people do not trust the site, they probably think the site provides fake information. Another survey shows that $31 \%$ of U.S. users are not at all acceptable about being shown messages from political campaigns on social media platforms. One survey shows that $54 \%$ of Americans are uncomfortable seeing political ads on social media platforms, and about $75 \%$ of Americans do not accept political campaigns using users' data to show them political ads [4].

All these surveys suggest that users relate political activities on social media platforms to one of the causes of platform distrust and misinformation. Most of the users feel uncomfortable when being recommended for political ads or posts. They know no matter how hard social media platform companies try to delete all the fake information on the platform, there will always be some existing. Even though the political campaigns on platforms are authentic, users are unwilling to engage in the campaign. Distrust between users and social media platform companies have increased since the inception of social media platforms and is not optimistic for the platform attracting more users and generating more network effects. Stand-alone utility for each user will be significantly reduced, then it will require larger additional utility that depends on the measure of participating users. So, platforms should avoid providing political ads and campaigns to increase the trust users have on platforms.

\section{SOCIAL ANXIETY}

A survey shows that around $50 \%$ of users feel positive after using the site, and another $50 \%$ of users feel negative after using it [10]. Social media users experience a mix of positive, negative emotions while using these platforms. A survey shows that roughly $40 \%$ of Americans have experienced online harassment [3]. They experience offensive name-calling, purposeful embarrassment, physical threats, sustained harassment, stalking and sexual harassment. Another survey shows that social media exerts severe negative feelings on teens. $24 \%$ of teenage users have a mostly negative effect that is harmful for their physical and mental health [2].

The major group of users that are often the victims of social anxiety and other negative symptoms are the teens who lack maturity of perceiving things between the truth and the fake. Many teens cannot stand online harassment from social media platform and choose to suicide, thus social anxiety from social media platforms becomes a major threat to teens' physical and mental health. Though many teens use social media platforms almost constantly, many teens quit using it. It will not bring a bright future to social media platform companies and will generate a negative within-group external effects. Therefore, social media platforms should create a separated platform for teens and children, setting strict regulations to remove all immoral content on the platform. But for people who are not teens but suffer from social anxiety, social media platforms should make tighter regulation on content to diminish social anxiety and online harassment.

\section{PLATFORM-BASESD ADVERTISEMENTS AND BUSINESSES}

A survey on Facebook ads shows that $47.4 \%$ of users learned about a new product or brand on Facebook, 26.9\% of users knew about the products or brand they saw on Facebook, and $25.7 \%$ of users did not see any ads on Facebook [11]. Another survey shows that $16.1 \%$ of Facebook users find ads relevant or interesting [11]. The rest of Facebook users do not find ads useful. However, a survey on Instagram ads shows a totally different story. $78 \%$ of Instagram users perceive brands on Instagram as popular, $77 \%$ of users perceive them as creative, $76 \%$ of users perceive them as entertaining, and $74 \%$ of users perceive them as relevant. $83 \%$ of users learn new products or services. $81 \%$ of users research products or services. $80 \%$ of users make decisions on whether to buy a product or service [1].

A significant amount of online transactions occur on social media platforms between users and platform-based advertisements and businesses. Many users now use social media platforms just to get more information on their favorite businesses in order to make a purchase. Two surveys on Facebook and Instagram both include the relevance of platform advertisements. It is clear to see that Instagram has done a better job than Facebook on providing more relevant products and services to their users. Whether platform-based advertisements and businesses will generate positive cross-group external effects, it largely depends on the relevance of advertisement with users. Social media platform companies should keep updating their algorithms on showing relevant advertisements to users. 


\section{CONCLUSION}

User experience plays a major role in creating network effects of social media platforms. Demographic differences in social media use let social media platform companies know what group of users they should target. Personal communication and news reading are two major things users do on social media platforms. Platforms should improve their service around these two major matters. User privacy is still a big concern for platform users. Once users are exposed to the risk of disclosing private information, it will be too late to fix the privacy policy and the positive within-group external effects will be largely reduced. Social media platforms should replace all false feed and information with accurate truth and facts. Social anxiety is a huge problem for users' mental and physical health, especially in teens. A large negative within-group external effects will be generated if social media platforms do not handle this well. Social media platforms should maintain the relationship between users, advertisers and businesses and keep up the relevance of ads provided to users. By doing so, more users will enter social media platforms to know more products and services from those advertisements and businesses. These analyses indicate that user experience is a rather complicated issue such that there are many more factors that restrain user experience, social media platform companies should pay careful attention to each of these factors that can affect network effects in several ways.

\section{REFERENCES}

[1] Aljohani, Mashael. Nisbet, Alastair. Blincoe, Kelly. (2016) A survey of social media user privacysettings $\&$ information disclosure.

[2] Anderson, Monica, and Jingjing Jiang. "Teens, Social Media \& Technology 2018." Pew Research Center: Internet, Science \& Tech, Pew Research Center, 31 May, 2018, www.pewresearch.org/internet/2018/05/31/teenssocial-media-technology-2018/.

[3] Auxier, Brooke, et al. "10 Tech-Related Trends That Shaped the Decade." Pew Research Center, 17 Aug. 2020, www.pewresearch.org/facttank/2019/12/20/10-tech-related-trends-thatshaped-the-decade/.

[4] Auxier, Brooke. "54\% Of Americans Say Social Media Companies Shouldn't Allow Any Political Ads." Pew Research Center, 24 Sept, 2020, www.pewresearch.org/fact-tank www.pewresearch.org/fact-tank/2020/09/24/54-ofamericans-say-social-media-companies-shouldntallow-any-political-ads/.

[5] "How Instagram Boosts Brands and Drives Sales."
Facebook IQ, 6 Feb. 2019, www.facebook.com/business/news/insights/howinstagram-boosts-brands-and-drives-sales.

[6] Perrin, Andrew, and Monica Anderson. "Share of U.S. Adults Using Social Media, Including Facebook, Is Mostly Unchanged since 2018." Pew Research Center, 10 Apr, 2019, www. PewResearch.org/fact-tank/2019/04/10/share-of-us-adults-using-social-media-including-facebook-ismostly-unchanged-since-2018/.

[7] Shearer, Elisa. Matsa, Katerina Eva. "News Use Across Social Media Platforms 2018." Pew Research Center's Journalism Project, 10 Sept, 2018, www.journalism.org/018/09/10/news-use-acrosssocial-media-platforms-2018/.

[8] Smith, Aaron. "Americans and Cybersecurity." Pew Research Center: Internet, Science \& Tech, Pew Research Center, 26 Jan, 2017, www.pewresearch.org/internet/2017/01/26/america ns-and-cybersecurity/.

[9] Raymond, Mark. "Social Media Usage Report 2019: User Habits You Need to Know." GoodFirms, www. goodfirms.co/resources/social-media-usage-userhabits-to-know.

[10] Sauro, Jeff. "The User Experience of Social Media Websites." MeasuringU, 20 Mar. 2018, measuringu.com/social-media-ux/.

[11] Zeibak, Leanna. "New Study: How Facebook Users Engage With Ads." Tinuiti, 23 Sept. 2019, tinuiti.com/blog/paid-social/facebook-consumerstudy-overview/. 